Dominic Voyer

Université du Québec à Rimouski, campus de Lévis

Michel Rousseau

Université du Québec à Trois-Rivières,

campus de Québec

Thomas Rajotte

Université du Québec à Rimouski, campus de Lévis

Viktor Freiman

Université de Moncton, campus de Moncton

Jim Cabot-Thibault

Commission scolaire René-Lévesque

\section{Le jeu d'échecs dans les classes du primaire : un moyen ciblé pour développer les habiletés des garçons en résolution de problèmes}

\author{
Chess in the classroom - a game targeted \\ to boys' problem-solving skills
}

doi: $10.18162 / f p .2018 .440$

\section{R \\ ésumé}

Dans cette recherche ${ }^{1}$, nous avons voulu vérifier si l'apprentissage du jeu d'échecs à lécole peut avoir un effet sur le développement des habiletés en résolution de problèmes mathématiques des garçons. Pour ce faire, pendant deux ans, nous avons donné des leçons à partir du contexte que procure le jeu d'échecs à 87 garçons du deuxième cycle du primaire. Des analyses répétées multiniveaux ont été menées à la fin de l'étude. Les résultats des analyses dégagent que les garçons ayant participé à l'intervention ont obtenu de meilleurs résultats en résolution de problèmes qu'un groupe témoin formé de 98 garçons.

\section{Mots-clés}

Résolution de problèmes mathématiques, apprentissage du jeu d'échecs, réussite des garçons.

Abstract

In this research, we wanted to test if learning and practicing chess game during school hours may have an effect on boys' mathematical problem solving skills. We have provided chess lessons for two years to 87 boys and 98 other boys took part of the control group. Multilevel repeated analyzes were conducted at the end of the study. Results show that boys who received chess lessons scored better in mathematical problems solving than boys from the control group.

Keywords Mathematical problem solving, chess learning, boys' achievement.

\section{Introduction}

Au courant des années 1990, à l'aube des travaux de la Commission des États généraux sur l'éducation, Baudelot et Establet (1992) encensaient la réussite québécoise en matière d'égalité des femmes et des hommes en éducation. Selon ces auteurs, le rendement toujours plus élevé des filles permettait au système scolaire canadien de se distinguer sur la scène internationale. Quelques années plus tard, la performance scolaire des filles surpassait celle des garçons dans l'ensemble des disciplines scolaires. En 2016, les données ministérielles ont même relevé que les garçons ont eu un plus faible rendement dans le domaine des mathématiques, une discipline pour laquelle ils obtenaient de manière récurrente de meilleurs résultats que les filles (Brochu, Deussing, Houme et Chuy, 2013; Ministère de l'Éducation et de l'Enseignement supérieur, 2016). La situation est d'autant plus préoccupante que le sentiment de compétences en mathématiques constitue une des caractéristiques dominantes permettant de prédire les risques de décrochage des élèves (Janosz et al., 2013).

Cet écart de rendement scolaire grandissant entre les garçons et les filles peut s'expliquer par différents facteurs. Parmi ceux-ci, le contexte de socialisation qui s'opère dans l'environnement scolaire et social des élèves est soulevé par certains auteurs (Benkirane, 2009; Royer, 2010). En effet, les garçons s'adapteraient moins bien aux règles strictes imposées à l'école (Royer, 2010; Thériault, 2006) et ils accorderaient plus d'importance au caractère inné de l'intelligence qu'à l'effort (Ministère de l'Éducation, 2004). Dans la classe, les garçons manifesteraient des intérêts différents de ceux des filles. La pression exercée par leur groupe de pairs les amènerait à développer des attitudes anticonformistes, qui perturberaient le climat scolaire (Lajoie, 2003; Thériault, 2006). Ils seraient généralement plus 
intéressés par les activités réalisées en groupe (Lemery, 2004), par les activités impliquant un certain degré de risque (Plante, Théorêt et Favreau, 2009), ainsi que par les activités à l'intérieur desquelles la compétition est présente (Lajoie, 2003).

Les pratiques enseignantes pourraient aussi contribuer aux inégalités scolaires et sociales entre les sexes (Lajoie, 2003; Plante et al., 2009). En effet, les attentes du milieu scolaire amèneraient les garçons et les filles à s'investir et à se conduire différemment en fonction de leur sexe. Plus particulièrement, les stéréotypes sexuels inciteraient les garçons à s'affirmer par des comportements nuisibles à leur réussite scolaire (Lajoie,2003; Royer,2010). Afin de régler partiellement ce problème, certains auteurs suggèrent de mettre en œuvre une pédagogie différenciée dans la classe. Ces auteurs soutiennent que la prise en considération des caractéristiques individuelles des garçons permet d'améliorer significativement leur réussite (Gurian, Stevens et King, 2008). Par exemple, des approches centrées sur l'expérimentation, plus kinesthésiques, faisant appel à des habiletés visuo-spatiales vont favoriser un meilleur rendement chez les garçons (King et Gurian, 2006).

Parmi les activités qui prennent en compte les caractéristiques et les intérêts des garçons, le jeu d'échecs semble être un outil pédagogique particulièrement intéressant, et ce, à différents égards. Historiquement, le jeu d'échecs a attiré majoritairement les garçons et cet engouement à l'égard de ce jeu perdure encore aujourd'hui (Blanch, Aluja et Cornadó, 2015). En effet, la Fédération internationale des échecs compte $93 \%$ de garçons parmi ses membres (Howard, 2005). Certains auteurs expliquent cet intérêt particulier qu'ont les garçons pour le jeu d'échecs en mentionnant le rôle de certains facteurs biologiques et environnementaux tels que les habiletés cognitives, le raisonnement mathématique ainsi que la possibilité d'actualiser ses habiletés visuo-spatiales (Blanch et al., 2015; Wai, Cacchio, Putallaz et Makel, 2010). De plus, par le contexte plus compétitif qu'il procure, le jeu d'échecs intéresse particulièrement les garçons (Bilalic, McLeod et Gobet, 2007). En plus d'engendrer un environnement positif à l'égard de l'apprentissage (Kovacic, 2012), ce contexte permet de développer la motivation scolaire (Scarano, 2014), le sentiment d'appartenance des élèves à l'égard de leur école ainsi que la qualité de la relation entre lélève et ses pairs (Rajotte, 2009). De plus, les garçons s'impliqueraient davantage lorsqu'une situation amène un certain niveau de risque, comme ce que propose une partie d'échecs (Grabner, Stern et Neubauer, 2007).

\section{Lejeu d'échecs et les habiletés en mathématiques}

Des études, provenant majoritairement du domaine de la psychologie, se sont intéressées aux habiletés cognitives des joueurs d'échecs. Certaines ont particulièrement étudié les habiletés en lien avec les mathématiques et ont conclu que le jeu d'échecs permet de favoriser la capacité à effectuer un raisonnement abstrait (Celone, 2001), à mettre en œuvre un raisonnement non verbal (Pearson, 2008), à développer des habiletés reliées à la numératie (Barrett et Fish, 2011) ou à développer les habiletés visuo-spatiales (Cabot-Thibault, 2013; Noir, 2002).

Par rapport à la résolution de problèmes, Ferreira et Palhares en 2008 ont voulu vérifier si des élèves de la troisième à la sixième année du primaire jouant dans un club d'échecs parascolaire obtiennent de meilleurs résultats en résolution de problèmes mathématiques que des élèves du même âge ne jouant pas à ce jeu. Leur étude a dégagé que les joueurs d'échecs performent mieux en résolution de problèmes que les élèves qui ne pratiquent pas cette activité. Ces auteurs émettent comme hypothèse qu'un 
enseignement du jeu d'échecs durant les heures de classe pourrait être bénéfique pour le développement des habiletés en résolution de problèmes mathématiques des élèves.

À ce sujet, plusieurs écoles offrent des cours d'échecs à leurs élèves. Par exemple, dans la province de Québec, certains organismes interviennent activement dans les écoles pour favoriser l'apprentissage à travers le jeu d'échecs. Ils offrent des ateliers de formation à quelque 2000 élèves de niveau primaire (6 à 12 ans) d'une soixantaine d'écoles réparties dans différentes régions de la province. Cependant, aucune étude longitudinale n'a été réalisée afin de vérifier si l'enseignement du jeu d'échecs durant les heures de classe a un effet positif sur les habiletés cognitives des élèves, notamment en résolution de problèmes. Plusieurs études sur le sujet ont plutôt comparé le rendement d'élèves pratiquant déjà le jeu d'échecs dans des clubs à des élèves ne jouant pas. Ces études montrent que les élèves fréquentant ces clubs d'échecs ont des habiletés mathématiques supérieures aux autres élèves (Ferreira et Palhares, 2008; Garcia, 2008). La question est maintenant de savoir si, en introduisant l'apprentissage et la pratique du jeu d'échecs dans le cursus scolaire, en remplaçant des périodes où la notion est travaillée par des activités à l'aide du jeu d'échecs, nous allons percevoir un effet significatif sur l'apprentissage de la résolution de problèmes mathématiques.

\section{Objectif et questions de recherche}

En prenant comme prémisse que le jeu d'échecs est adapté aux caractéristiques et aux intérêts des garçons et en nous appuyant sur les études qui suggèrent que le jeu d'échecs peut avoir des effets positifs sur le rendement des élèves dans les matières scolaires, nous avons voulu mettre à l'épreuve l'hypothèse, notamment énoncée par Ferreira et Palhares (2008), selon laquelle l'apprentissage et la pratique du jeu d'échecs en classe peuvent permettre de solliciter et développer les habiletés en résolution de problèmes mathématiques des garçons. Nous avons ciblé la résolution de problèmes, d'une part parce que cette compétence est au cœur des programmes en éducation et d'autre part parce que le jeu d'échecs pourrait, de par les liens qu'il exige de faire, favoriser son développement.

La présente étude vise donc à répondre à la question suivante : "Dans quelle mesure l'apprentissage du jeu d'échecs dans le cadre scolaire peut-il favoriser le développement des habiletés en résolution de problèmes mathématiques des garçons?"

\section{Contexte théorique}

\section{La résolution de problèmes mathématiques}

La résolution de problèmes occupe une place importante dans les curriculums scolaires depuis les années 1980 et son importance n'est plus à démontrer même s'il existe des différences sémantiques à son égard dans les ouvrages scientifiques (Martin et Theis, 2009). Le Programme de formation de l'école québécoise en a fait l'une des trois compétences à développer pour la discipline mathématique et le National Council of Teachers of Mathematics (NCTM) (2000) la présente comme l'un de ses principes fondamentaux. 
La résolution de problèmes se voit attribuer une double finalité dans les curriculums scolaires. Elle est vue à la fois comme une habileté à développer et comme un moyen pour développer d'autres connaissances (Freiman et Savard, 2014). Il y a donc l'enseignement de la résolution de problèmes et l'enseignement par la résolution de problèmes (Voyer et Goulet, 2013).

La résolution de problèmes est un processus [cognitif] complexe comprenant plusieurs étapes. Au cours des 70 dernières années, plusieurs chercheurs ont produit des définitions et des schématisations de ce processus complexe (par exemple : Poirier, 2001; Pólya, 1945; Reusser, 1990; Verschaffel, Greer et De Corte, 2000). Le modèle développé par Verschaffel et al. (2000) comprend les étapes suivantes : compréhension de la situation, construction d'un modèle mathématique, résolution à l'aide du modèle mathématique, interprétation du résultat en fonction de la compréhension de la situation et communication du résultat. Voici une schématisation de ce processus :

251663360251664384251668480251673600251675648251676672

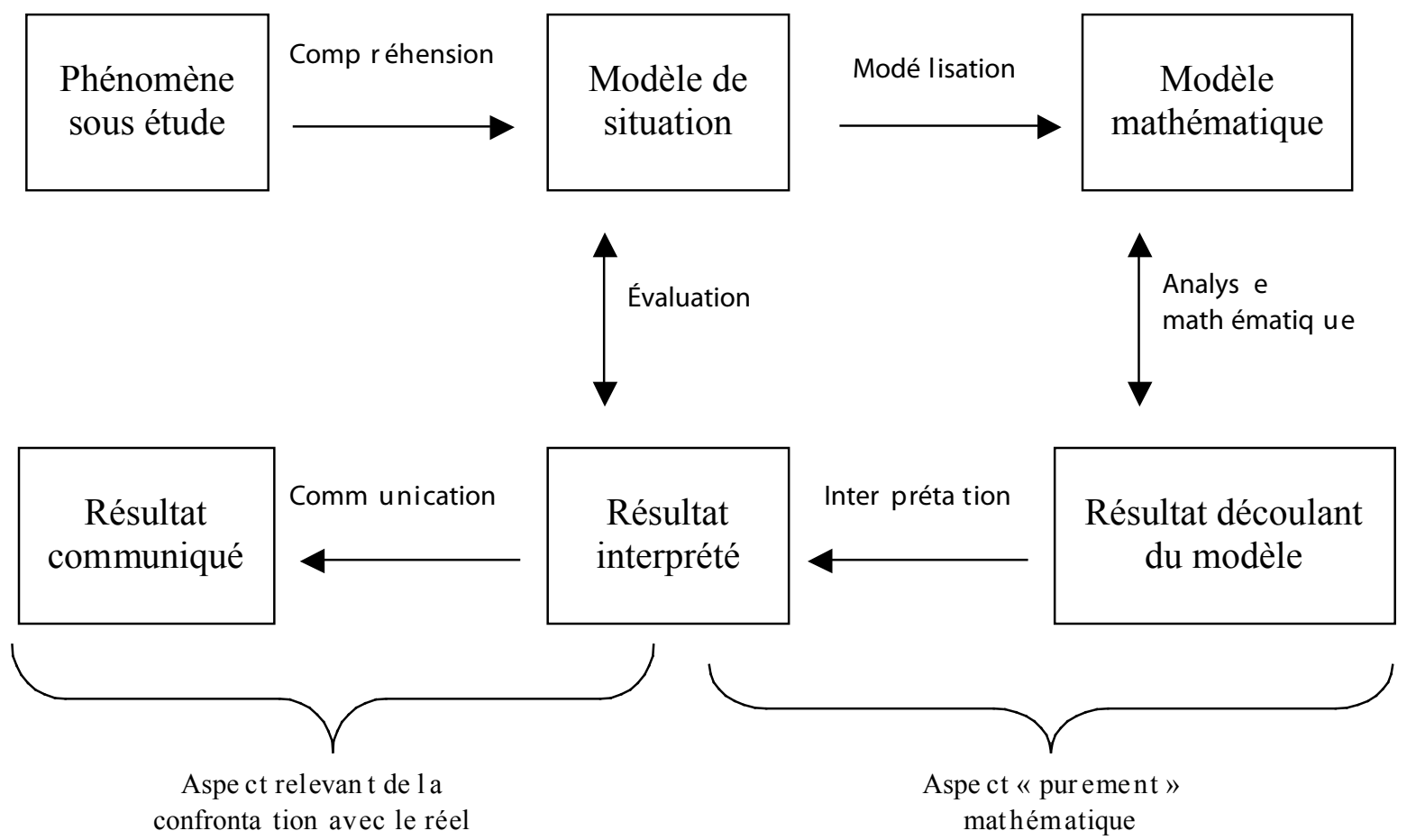

Figure 1

Processus de résolution de problèmes de Verschaffel et al. (2000) traduit par Fagnant, Demonty et Lejong (2003) 
Il est à noter que ce processus de résolution de problèmes doit être vu de façon circulaire plutôt que linéaire. Dépendamment de la complexité du problème, des aller-retour entre certaines étapes peuvent survenir. Par exemple, la compréhension initiale du problème peut mener le solutionneur à un modèle mathématique impossible à mettre en place en raison de l'absence de certaines données. À ce moment, le solutionneur retournera à l'étape de la compréhension pour générer un nouveau modèle mathématique.

\section{Le jeu d'échecs et la résolution de problèmes}

Le jeu d'échecs peut être considéré comme un moyen d'enseigner la résolution de problèmes. Dans sa thèse, Noir (2002) établit la relation entre la résolution de problèmes échiquéens et la résolution de problèmes mathématiques de la manière suivante :

le problème d'échecs s'aborde comme un problème mathématique : analyse des données (les pièces sur l'échiquier, leurs positions, les menaces, protections et combinaisons), énoncé des hypothèses et simulation des coups possibles en déduction, plan logique à suivre [...] Si l'enseignement des mathématiques a pour but essentiel de doter l'enfant d'une capacité de raisonnement et de méthode, le jeu d'échecs est sans aucun doute celui qui peut développer le mieux ses facultés dans ce domaine (p. 4)

Cette relation décrite par Noir (2002) peut être reprise en utilisant le processus de résolution de problèmes de Verschaffel et al. (2000). Pour ce faire, voici un exemple de problème échiquéen pour joueur débutant.

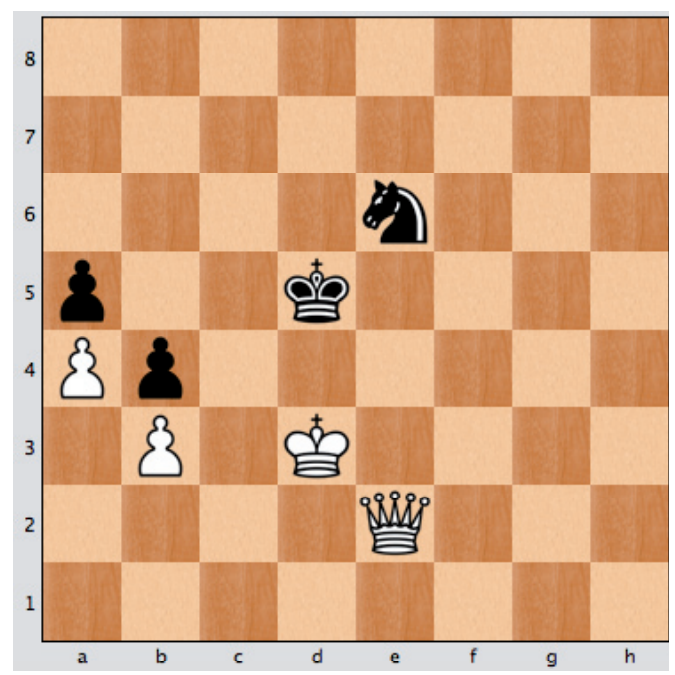

Figure 2

Exemple de problème échiquéen pour débutant : "Trouve le coup des noirs qui permet d'attaquer deux pièces blanches simultanément » 


\section{Tableau 1}

Légende des pièces du jeu d'échecs

\begin{tabular}{|l|l|}
\hline Pièce du jeu d'échecs & Représentation \\
\hline Cavalier & \\
\hline Pion & \\
\hline Dame & \\
\hline Roi & \\
\hline
\end{tabular}

Dans le problème présenté, le joueur doit d'abord analyser et comprendre la position présentée. Dans ce cas-ci, il sait que c'est aux pièces noires de jouer et qu'il doit trouver une façon de bouger une pièce pour que celle-ci attaque deux pièces blanches (étape de la compréhension). Par la suite, il utilise ses «savoirs échiquéens» (connaissance des règles et de stratégies) pour tenter de trouver la solution (construction d'un modèle mathématique et résolution à l'aide de ce modèle ${ }^{2}$ ). Dans l'exemple, le joueur sait, par sa connaissance des règles, que les deux pions noirs situés en a 5 et en b4 ne peuvent pas bouger étant bloqués par les pions blancs en b3 et a4. Il pourrait tenter une première solution : bouger son roi en c5. Ce mouvement du roi est permis dans la position, mais lorsque l'on interprète ce coup en fonction de la compréhension du problème, on voit que ce coup de roi ne permet pas d'attaquer deux pièces blanches simultanément (interprétation de la solution en fonction de la compréhension). Cela renvoie donc le joueur à l'étape de construction d'un modèle "échiquéen ». En analysant les coups possibles par le cavalier et en référant à la stratégie de la « fourchette », l'élève bouge son cavalier en f4. Il interprète de nouveau ce coup en fonction de sa compréhension du problème. Comme le roi blanc en d3 et la dame blanche en e2 sont attaqués, cette solution respecte les contraintes du problème. L'élève peut alors communiquer cette solution verbalement, par écrit ou directement sur l'échiquier.

\section{Méthodologie}

\section{Participants}

Les participants à l'étude provenaient de 11 écoles francophones de la province de Québec. Léchantillon utilisé pour les analyses était composé de 186 garçons de 8-9 ans, soit 87 pour le groupe expérimental et 98 pour le groupe témoin. L'ensemble des écoles impliquées provenait de la Commission scolaire des Chênes. La répartition des écoles au sein des groupes contrôle et expérimental a été effectuée de manière à assurer une homogénéité des niveaux socioéconomiques entre les deux groupes de l'étude. À cet effet, l'indice de milieu socioéconomique et l'indice du seuil de faible revenu tels que rapportés par le ministère de l'Éducation et de l'Enseignement supérieur ont été utilisés afin de former les deux groupes participant à l'étude. Cette considération visait essentiellement à rendre les groupes plus homogènes puisque dans la commission scolaire participante il y avait des différences entre les écoles à cet égard. 


\section{Déroulement de l'étude}

L'étude a été réalisée sur une période de deux années scolaires. Lors de l'automne de la première année, alors que les participants venaient d'entamer leur troisième année du primaire, la première prise de mesures a été effectuée. À la suite de cette première prise de mesure, les participants du groupe expérimental ont reçu des leçons du jeu d'échecs en classe à raison d'une heure par semaine pendant 30 semaines par année pour un total de 60 heures de cours pour les deux années de l'étude. Durant cette période, tous les participants ont été soumis à trois autres prises de mesure qui ont eu lieu à la fin de la première année, au début de la deuxième et à la fin de la deuxième. Ces prises de mesure ont permis de suivre l'évolution des participants à un test de résolution de problèmes mathématiques sur les deux années du projet.

\section{Déroulement et contenu des leçons}

Les 60 heures de leçons du jeu d'échecs ont été offertes en collaboration avec l'Académie d'échecs. Il s'agit d'un organisme à but non lucratif dont la mission est de promouvoir l'apprentissage et la pratique du jeu d'échecs à l'école. Des guides d'apprentissage produits par cet organisme ont aussi été utilisés comme support lors des leçons. Chacune des leçons était divisée en trois parties. La première partie permettait de faire une révision des concepts vus lors de la leçon précédente. Par la suite, un nouveau concept était présenté et des problèmes étaient proposés aux élèves. Finalement, les élèves étaient invités à jouer une partie. Cela se déroulait sous la supervision de l'instructeur qui amenait les élèves à réfléchir sur leur partie et à effectuer des liens avec la théorie présentée préalablement.

Pour ce qui est du contenu, l'ensemble des phases du jeu d'échecs a été abordé à travers les leçons : le fonctionnement des pièces, les ouvertures, le milieu de partie, la tactique et les finales. Étant donné le temps disponible et l'âge des participants, il était utopique de viser l'appropriation de tous les éléments du jeu par les élèves, mais réaliste de leur faire découvrir. De nombreux problèmes semblables à celui présenté à la figure 2 ont été présentés aux élèves tout au long de l'expérimentation.

\section{Devis de recherche et variables impliquées dans l'étude}

Afin d'opérationnaliser ce projet de recherche, un devis quasi expérimental impliquant une série temporelle multiple a été utilisé. Il aurait été irréaliste d’opter pour une répartition aléatoire des participants à l'intérieur des groupes expérimental et témoin. Il nous fallait travailler avec différents groupes dans leur classe respective.

La participation (ou non) au programme scolaire d'enseignement des échecs correspondait à la variable indépendante de l'étude. C'est cette variable qui était manipulée par le chercheur. Ensuite, les habiletés en résolution de problèmes mathématiques correspondaient à la variable dépendante de la recherche. C'est cette variable qui, selon nos hypothèses, devait recevoir l'effet présumé de la variable indépendante. 


\section{Le test utilisé}

Pour mesurer l'habileté à résoudre des problèmes mathématiques, nous avons administré aux élèves, à chacun des temps de mesure, huit problèmes mathématiques. Les problèmes choisis étaient du type "problèmes verbaux» (word problems). Selon Verschaffel et De Corte (2008), ces problèmes sont utilisés pour «[...] développer chez les élèves la capacité de savoir quand et comment appliquer les mathématiques de manière efficace $[. .] ».($ p. 154). Ces auteurs mentionnent également que ce type de problème sollicite la mise en œuvre d'un processus de résolution de problèmes tel que celui décrit à la figure 1. Comme l'étude s'est déroulée sur une période de deux années scolaires, les problèmes utilisés ont dû être adaptés en fonction du niveau scolaire en mathématiques des élèves. Par exemple, les problèmes utilisés au temps 1 étaient adaptés à des élèves commençant leur troisième année du primaire alors que ceux utilisés au temps 4 étaient adaptés à des élèves terminant leur quatrième année. Les problèmes ont été choisis parmi une banque d'instruments de mesure de la société GRICS. Cette banque de problèmes est habituellement utilisée par les écoles et les enseignants pour produire des tests de mathématiques. Les problèmes retenus n'avaient aucun lien avec le jeu d'échecs. De plus, les problèmes sélectionnés relevaient des principaux domaines des mathématiques associés au curriculum scolaire du primaire, soit : l'arithmétique, la géométrie ainsi que les probabilités et statistiques. Les problèmes administrés ont permis de créer une variable intitulée "rendement en résolution de problèmes ".

\section{Équilibrage des échelles}

Puisque les problèmes qui composaient les versions utilisées à chacun des temps de mesure étaient adaptés au niveau scolaire des élèves, le niveau de difficulté d'un test à l'autre pouvait varier et risquait d'avoir pour effet que les différences observées soient des différences reliées au niveau de difficulté variable de chacune des versions. Pour pouvoir comparer les résultats du test à chacune des prises de mesure, certains items étaient les mêmes d'une version à l'autre du test, ces items sont appelés items ancrés. Cette procédure permet l'équilibrage des échelles (test equating). Léquilibrage des échelles a pour but de contrôler la différence dans le niveau de difficulté global de chaque version en situant les scores des versions utilisées aux temps 2, 3 et 4 par rapport au niveau de difficulté de la version utilisée au temps 1. Pour les quatre prises de mesure, 26 problèmes différents ont été utilisés au total. Pour les prises de mesure 2, 3 et 4, les tests contenaient deux problèmes présentés lors de la mesure précédente (les items ancrés) et six nouveaux problèmes. L'équilibrage des échelles a été réalisé à l'aide du modèle de Rasch. Les items ancrés ont été utilisés pour permettre de situer les scores des versions des prises de mesure 2, 3 et 4 sur la même échelle de valeurs que la version du temps 1. Ces analyses ont été réalisées à l'aide du logiciel Winsteps.

\section{Sources d'invalidité contrôlées par le devis}

Le devis utilisé, avec quatre prises de mesure et le recours à un groupe témoin, a permis de contrôler différents biais susceptibles d'influer sur l'analyse des données. Dans un premier temps, l'insertion d'un groupe témoin dans le protocole de l'étude a permis de contrôler la source d'invalidité interne associée aux facteurs historiques des participants (Vallerand et Hess, 2000). En effet, bien que certaines 
expériences vécues par les participants en dehors de l'intervention mise en place aient pu influencer le rendement aux tests, ces expériences devraient se répartir équitablement entre les participants des deux groupes (Ladouceur et Bégin, 1980). Pour ce projet, cela pourrait se traduire par des activités parascolaires de certains élèves, par des pratiques pédagogiques particulières des enseignants (nous avions 11 enseignants) ou simplement par le projet éducatif de l'établissement scolaire.Conséquemment, afin de nous assurer de la meilleure validité de nos conclusions ainsi que des interprétations qui en découlent, nous devions nous assurer de mettre en place un devis permettant de contrôler, au mieux, les sources d'invalidité.

En utilisant une série temporelle multiple, c'est-à-dire en effectuant plusieurs prises de mesure auprès des participants du groupe expérimental et du groupe témoin, le devis de recherche utilisé a permis de contrôler les sources d'invalidité liée aux expériences antérieures qu'ont les participants avant d'entreprendre le programme d'intervention et à la réactivité à l'égard de l'instrument de mesure (Alain, Pelletier et Boivin, 2000). En effet, bien que les deux groupes nétaient pas équivalents (puisqu'il est très difficile d'effectuer une répartition aléatoire des participants dans le cadre d'une recherche réalisée dans des écoles), la première prise de mesure effectuée a permis de situer le rendement en résolution de problèmes avant d'amorcer l'expérimentation, et ce, afin de comparer l'évolution de cette variable dans les deux groupes tout au long des deux années de l'intervention. De plus, le devis de recherche a aussi permis de contrôler le biais lié à la réactivité à l'égard de l'instrument de mesure. En effet, bien que nous ayons procédé à un équilibrage des échelles et que l'ancrage d'items lors des prises de mesure 2, 3 et 4 puisse avoir influencé le rendement de l'élève en le familiarisant à l'égard de certains des énoncés utilisés, cette influence possible devrait se traduire équitablement dans les groupes témoin et expérimental.

\section{Analyse}

Les données manquantes constituent un problème pour la majorité des études longitudinales. Pour la présente étude, les quatre prises de mesure ont été complétées par 109 garçons, ce qui représente $58,6 \%$ de l'échantillon. Parmi les garçons ayant des résultats incomplets, 23 (12,4\%) ont participé à 3 temps de mesure et $53(28,5 \%)$ à deux temps de mesure.

La plupart des méthodes d'analyses de données comme les ANOVA à mesures répétées ont besoin de toutes les prises de mesure pour fonctionner. C'est donc dire que tous les garçons n'ayant pas répondu à tous les temps de mesure devaient être retirés systématiquement. Cela entraine une perte de puissance statistique et un biais (Allison, 2001). Pour éviter ce problème, il est possible d'utiliser un modèle de régression multiniveaux pour données longitudinales. Dans ce contexte, les données longitudinales peuvent être représentées comme étant des mesures répétées pour chaque élève. Un aspect intéressant de ce modèle est qu'il n'exige pas nécessairement qu'un élève ait participé à toutes les prises de mesure pour être considéré dans l'analyse. Le modèle utilise plutôt les informations de la base de données pour estimer les paramètres du modèle (Hox, 2000). 
Les analyses de régression multiniveaux effectuées ont examiné trois modèles distincts. Le modèle 1, correspondant au modèle de base, inclut uniquement les scores au test obtenus à chacun des quatre temps pour tous les participants. Ce modèle permet de tester si le score en mathématiques change d'une année à l'autre et d'évaluer l'importance de ce changement. Pour le deuxième modèle, la variable " temps » est ajoutée comme prédicteur des mesures répétées. Ce modèle permet d'estimer si le changement dans les scores peut être expliqué par le temps de mesure et si ce changement varie d'un élève à l'autre. En d'autres termes, ce modèle permet de vérifier s'il y a un accroissement linéaire pour chacun des garçons dans le temps et si cet accroissement est variable d'un garçon à l'autre. Si l'accroissement est similaire pour tous les garçons de l'échantillon, le traitement expérimental ne pourra pas avoir eu d'effet. Pour le modèle 3, la variable " groupe » (expérimental ou témoin) a été ajoutée afin de savoir si la variation de l'accroissement des scores en résolution de problèmes mathématiques dépendait du fait d'avoir participé au programme du jeu d'échecs ou non. C'est donc ce troisième modèle qui permet de répondre précisément à notre question de recherche. Les analyses ont été effectuées avec le logiciel STATA/SE version 14.2. La méthode de maximum de vraisemblance a été utilisée pour estimer les paramètres du modèle.

\section{Résultats}

La moyenne au temps 1 des élèves du groupe expérimental est similaire à celle du groupe contrôle. Il y a aussi peu de différence observée entre les moyennes des deux groupes au temps 2. Cependant, lors du temps 3 et 4 , les élèves du groupe expérimental ont une moyenne supérieure à celle des élèves du groupe contrôle. Bien que le nombre d'élèves varie d'un moment à l'autre du devis, une certaine progression est observée entre la mesure au temps 1 et celle au temps 4 suggérant que les élèves ont amélioré leur compétence en résolution de problèmes entre le début et la fin de cette étude.

\section{Tableau 2}

Résultats descriptifs

\begin{tabular}{|c|c|c|c|c|c|c|c|c|}
\hline & \multicolumn{2}{|r|}{ Temps 1} & \multicolumn{2}{|r|}{ Temps 2} & \multicolumn{2}{|r|}{ Temps 3} & \multicolumn{2}{|r|}{ Temps 4} \\
\hline & $n$ & $M$ & $\mathrm{n}$ & $M$ & $\mathrm{n}$ & M & $n$ & $M$ \\
\hline & & (É.T.) & & (É.T.) & & (É.T.) & & (É.T.) \\
\hline Expérimental & 79 & $45,0(19,1)$ & 81 & $62,2(23,6)$ & 74 & $68,6(23,6)$ & 70 & $89,4(16,5)$ \\
\hline Contrôle & 76 & $44,2(18,3)$ & 81 & $62,1(19,6)$ & 75 & $59,5(23,5)$ & 73 & $83,7(16,9)$ \\
\hline Total & 156 & $44,6(18,6)$ & 162 & $62,2(21,6)$ & 150 & $64,1(23,9)$ & 144 & $86,5(16,9)$ \\
\hline
\end{tabular}

\section{Modèle 1}

Ce modèle départage la variance totale des scores aux tests en deux types de variance, la variance liée aux différences entre les temps de mesure et la variance liée aux différences entre les élèves. La composante de variance liée aux mesures répétées est de 500,7 et la composante de variance liée aux différences entre les élèves est de 129,9. Ainsi, la proportion de variance liée aux différences entre les élèves est estimée à $21 \%$. Cela signifie que la variance des scores aux tests est expliquée au quatre cinquième par la variation des scores dans le temps et au un cinquième par les différences entre les 
garçons. La valeur de l'intercept du modèle fait référence à la moyenne générale au test pour l'ensemble des 4 temps de mesure, soit 63,3.

\section{Modèle 2}

Le facteur temps étant statistiquement significatif, cela signifie qu'un modèle linéaire d'augmentation des scores aux tests de résolution de problèmes permet d'expliquer les différences entre les temps de mesure. Ce modèle linéaire prédit qu'en moyenne les élèves obtiennent 44,4 points au premier temps de mesure. Ce score augmente ensuite en moyenne de 12,8 points à chacun des autres temps de mesure consécutifs.

\section{Modèle 3}

Dans ce dernier modèle, la variable "groupe » a été ajoutée pour vérifier si les élèves du groupe expérimental présentent un accroissement plus élevé que ceux du groupe contrôle. Les résultats montrent un effet significatif de cette variable. Le dernier modèle prédit qu'en moyenne, les élèves obtiendront un score de 42,2 points lors du premier temps de mesure. Par la suite, ils augmenteront en moyenne de 12,9 points à chaque temps de mesure. Pour les élèves du groupe expérimental, ceux-ci verront leur accroissement moyen de 4,3 points de plus que ceux du groupe contrôle.

Les garçons du groupe expérimental ont augmenté de 4,3 points de plus que le groupe témoin à chacun des temps de mesure $(\mathrm{p}<0,05)$.

\section{Tableau 3}

Paramètres du modèle multiniveaux

\begin{tabular}{llll}
\hline & Intercept & Temps & Groupe \\
\hline Modèle 1 & $63,3(1,2)$ & - & - \\
Modèle 2 & $44,4(1,5)$ & $12,8(0,6)$ & \\
Modèle 3 & $42,2(1,9)$ & $12,9(0,6)$ & $4,3(2,4)$ \\
\hline
\end{tabular}

\section{Discussion des résultats}

Les résultats montrent que l'apprentissage du jeu d'échecs en milieu scolaire peut favoriser le développement des habiletés en résolution de problèmes mathématiques des garçons. Sur une perspective longitudinale, les garçons participant ou non au programme scolaire d'enseignement des échecs bénéficient des pratiques courantes d'enseignement et développent leurs habiletés en résolution de problèmes à l'école. Nous avons cependant constaté que l'apprentissage du jeu d'échecs en milieu scolaire permet de constituer un complément signifiant à l'enseignement usuel de la résolution de problèmes au primaire. Ce constat se justifie par le fait qu'après deux ans, les garçons du groupe expérimental obtiennent un rendement en résolution de problèmes plus élevé que celui observé chez les garçons du groupe témoin. 
Ces résultats viennent appuyer l'hypothèse avancée par Ferreira et Palhares (2008) selon laquelle un enseignement du jeu d'échecs en classe est bénéfique pour le développement des habiletés en résolution de problèmes des élèves. Aussi, ces résultats appuient l'initiative des écoles à offrir des leçons du jeu d'échecs durant les heures de mathématiques au primaire.

Nous expliquons ce résultat par le fait que le jeu d'échecs présente des conditions de résolution de problèmes véritables qui se rapprochent de ce qui est attendu en classe de mathématiques lors d'activités de résolution de problèmes. En effet, tel que présenté dans le contexte théorique, le processus de résolution de problèmes mathématiques peut être utilisé pour résoudre des problèmes échiquéens. Des études complémentaires, possiblement qualitatives, pourraient maintenant mieux étudier la façon dont la résolution de problèmes échiquéens contribue aux habiletés en résolution de problèmes mathématiques. Il serait notamment pertinent de s'intéresser à l'installation et à la rétention des effets que procure le jeu d'échecs sur la résolution de problèmes mathématiques. Nos résultats montrent qu'un temps d'installation a été nécessaire avant de percevoir les effets aux temps 3 et 4. Se pourrait-il que la phase d'apprentissage liée au contexte du jeu (fonctionnement de l'échiquier, règles de déplacement des pièces, etc.) ait été un investissement à plus long terme? Qu’en serait-il d'une utilisation du jeu d'échecs plus soutenue à plus long terme? Autant de questions auxquelles nous ne pouvons répondre pour l'instant mais qui ouvrent à de nouvelles études.

Nos résultats appuient l'idée que le jeu d'échecs pourrait être utilisé comme moyen pédagogique complémentaire aux activités habituelles de résolution de problèmes vécues en classe, particulièrement chez les garçons. Afin de maximiser le potentiel d'apprentissage de la résolution de problèmes par le jeu d'échecs, l'enseignant pourrait expliciter aux élèves ce parallèle entre résoudre un problème mathématique et résoudre un problème échiquéen. En plus de l'aspect motivationnel relaté par les enseignants que permet d'engendrer le jeu d'échecs, il s'agit d'un contexte qui permet de résoudre des problèmes sans faire intervenir l'habileté en lecture, celle-ci pouvant être une embuche supplémentaire dans l'apprentissage de la résolution de problèmes, et ce, particulièrement pour les garçons. Le jeu d'échecs nous apparait donc comme un contexte pédagogique différent et pertinent pour amener la résolution de problèmes en classe, un contexte qui est par ailleurs bien adapté aux garçons.

\section{Conclusion}

$\mathrm{Au}$ Québec, les récentes données ministérielles ont démontré que les garçons accusent maintenant un retard dans l'ensemble des disciplines scolaires par rapport aux filles, et ce, même en mathématiques, un domaine à l'intérieur duquel les garçons se distinguaient auparavant (Brochu et al., 2013; Ministère de l'Éducation et de l'Enseignement supérieur, 2016). Certaines études avancent que cette situation peut être attribuable au fait que l'école ne soit pas adaptée aux caractéristiques des garçons telles que la compétitivité, le gout du risque et l'attrait pour les activités kinesthésiques. À ce sujet, le jeu d'échecs rejoint les caractéristiques des garçons. Malheureusement, peu de données sérieuses sont disponibles pour évaluer les effets de l'utilisation du jeu d'échecs en classe. Par cet article, nous contribuons à montrer que l'apprentissage et l'utilisation du jeu d'échecs en classe peut permettre de développer des habiletés en résolution de problèmes mathématiques chez les garçons. Considérant le rôle prépondérant des habiletés en mathématiques dans la réussite scolaire, nous voyons dans le jeu d'échecs un outil pédagogique à exploiter. Nous pensons qu'il serait possible de tirer profit du potentiel d'apprentissage 
de cet outil en valorisant la mise en ouvre d'interventions se rapportant à l'utilisation du jeu d'échecs dans le milieu scolaire, et ce, pour remplacer des activités habituelles. Certains manuels scolaires ont déjà exploité cette avenue par le passé, mais d'une façon très ponctuelle. Une utilisation plus soutenue pourrait selon nous favoriser le rendement des garçons en mathématiques et par conséquent contribuer à la qualité du lien affectif qu'ils entretiennent avec l'école. Dans cette même voie, une recension de stratégies d'enseignement alternatives de la résolution de problèmes et d'autres notions qui seraient mieux adaptées aux garçons pourrait selon nous être une suite intéressante au travail amorcé ici.

\section{Notes}

1 Cette étude a été financée par le Conseil de recherches en sciences humaines du Canada.

2 Dans le cas d'une partie d'échecs, le « modèle mathématique » sera bâti à l'aide des savoirs « échiquéens " plutôt que mathématiques.

\section{Références}

Alain, M., Pelletier, L. G. et Boivin, M. (2000). Les plans de recherche expérimentaux. Dans R. J. Vallerand et U. Hess (dir.), Méthodes de recherche en psychologie (p. 137-170). Boucherville, QC : Gaëtan Morin.

Allison, P. D. (2001). Missing data: a Sage University papers series on quantitative applications in the social sciences $\left(\mathrm{n}^{\circ} 07-136\right)$. Thousand Oaks, CA : Sage.

Barrett, D. C. et Fish, W.W. (2011). Our move: Using chess to improve math achievement for students who receive special education services. International Journal of Special Education, 26(3), 181-193. Repéré à http://files.eric.ed.gov/fulltext/EJ959011.pdf

Baudelot, C. et Establet, R. (1992). Allez les filles!. Paris : Seuil.

Benkirane, K. (2009). Culture de la masculinité et décrochage scolaire des garçons au Québec. Paris : L'Harmattan.

Bilalic, M., McLeod, P. et Gobet, F. (2007). Personality profiles of young chess players. Personality and Individual Differences, 42(6), 901-910. http://dx.doi.org/10.1016/j.paid.2006.08.025

Blanch, A., Aluja, A. et Cornadó, M.-P. (2015). Sex differences in chess performance: Analyzing participation rates, age, and practice in chess tournaments. Personality and Individual Differences, 86, 117-121.

http://dx.doi.org/10.1016/j.paid.2015.06.004

Brochu, P., Deussing, M.-A., Houme, K. et Chuy, M. (2013). À la hauteur : Résultats canadiens de l'étude PISA de l'OCDE. Le rendement des jeunes du Canada en mathématiques, en lecture et en sciences : premiers résultats de 2012 pour les jeunes du Canada âgés de 15 ans. Repéré à http://cmec.ca/Publications/Lists/Publications/Attachments/318/PISA2012 CanadianReport fr Web.pdf

Cabot-Thibault, J. (2013). L'effet de l'apprentissage du jeu d'échecs dans le cadre scolaire sur le développement du sens spatial d'élèves du premier cycle du secondaire (Mémoire de maitrise, Université du Québec à Rimouski). Repéré à http://semaphore.uqar.ca/939

Celone, J. (2001). The effects of a chess program on abstract reasoning and problem-solving in elementary school children: $A$ thesis submitted to the school of graduate studies in partial fulfilment of the requirement for the degree of master of science (Mémoire de maitrise). Southern Connecticut State University, New Haven, CT.

Fagnant, A., Demonty, I. et Lejong, M. (2003). La résolution de problèmes : un processus complexe de « modélisation mathématique ». Informations pédagogiques, 54, 29-39.

Ferreira, D. et Palhares, P. (2008). Chess and problem solving involving patterns. The Mathematics Enthusiast, 5(2-3), 249256. Repéré à http://scholarworks.umt.edu/tme/vol5/iss2/8 
Freiman, V. et Savard, A. (2014). Résolution de problèmes en mathématiques. Éducation et francophonie, 42(2), 1-6. http://dx.doi.org/10.7202/1027902ar

Garcia, N. V. (2008). Scholastic chess club participation and the academic achievement of hispanic fifth grade students in south Texas (Thèse de doctorat). Accessible par ProQuest Dissertations \& Theses. (3309547)

Grabner, R. H., Stern, E. et Neubauer, A. C. (2007). Individual differences in chess expertise: A psychometric investigation. Acta Psychologica, 124(3), 398-420. http://dx.doi.org/10.1016/j.actpsy.2006.07.008

Gurian, M., Stevens, K. et King, K. (2008). Strategies for teaching boys and girls: Secondary level. San Francisco, CA : JosseyBass.

Howard, R. W. (2005). Are gender differences in high achievement disappearing? A test in one intellectual domain. Journal of Biosocial Science, 37(3), 371-380. http://dx.doi.org/10.1017/S0021932004006868

Hox, J. J. (2000). Multilevel analysis of grouped and longitudinal data. Dans T. D. Little, K. U. Schnabel et J. Baumert (dir.), Modeling longitudinal and multilevel data: Practical issues, applied approaches, and specific examples (p. 15-32). Mahwah, NJ : Lawrence Erlbaum.

Janosz, M., Pascal, S., Belleau, L., Archambault, I., Parent, S. et Pagani, L. (2013). Les élèves du primaire à risque de décrocher : caractéristiques à 12 ans et prédicteurs à 7 ans - Étude longitudinale du développement des enfants du Québec (ÉLDEQ 1998-2010). Québec, QC : Institut de la statistique du Québec.

King, K. et Gurian, M. (2006). With boys in mind / Teaching to the minds of boys. Educational Leadership, 64(1), 56-61. Repéré à http://www.ascd.org/publications/educational-leadership/sept06/vol64/num01/Teaching-to-the-Minds-of-Boys.aspx

Kovacic, D. M. (2012). Ajedrez en las escuelas. Una buena movida. Psiencia, revista latinoamericana de ciencia psicológica, 4(1), 29-41. Repéré à http://www.psiencia.org/ojs/index.php/psiencia/article/view/87

Ladouceur, R. et Bégin, G. (1980). Protocoles de recherche en sciences appliquées et fondamentales. Saint-Hyacinthe, QC : EDISEM.

Lajoie, G. (2003). L'école au masculin : réduire l'écart de réussite entre garçons et filles. Québec, QC : Septembre.

Lemery, J.-G. (2004). Les garçons à l'école : une autre façon d'apprendre et de réussir. Montréal, QC : Chenelière McGraw-Hill.

Martin, V. et Theis, L. (2009). Les élèves à risque au cœur d'une activité de résolution de problèmes : l'exemple des probabilités. Dans Actes du Colloque International Espace Mathématique Francophone (p. 1119-1129). Repéré à http://fastef.ucad.sn/EMF2009/Groupes\%20de\%20travail/GT8/Microsoft\%20Word\%20\%20Martin\%20et\%20Theis-EMF2009.pdf

Ministère de l'Éducation. (2004). La réussite des garçons : des constats à mettre en perspective. Rapport synthèse. Repéré à http://www.education.gouv.qc.ca/fileadmin/site web/documents/dpse/reussite garcon.pdf

Ministère de l'Éducation et de l'Enseignement supérieur. (2016). Examens et épreuves : tableau 4 - résultats par matière, selon certaines variables, pour la session de juin 2014. Québec, QC : Ministère de l'Éducation et de l'Enseignement supérieur.

National Council of Teachers of Mathematics (NCTM). (2000). Principles and standards for school mathematics. Reston, VA : NCTM.

Noir, M. (2002). Le développement des habiletés cognitives de l'enfant par la pratique du jeu d'échecs : essai de modélisation d'une didactique du transfert (Thèse de doctorat, Université Lumière Lyon 2). Repéré à http://www.theses.fr/2002LYO20023

Pearson, T. R. (2008). The effects of learning to play chess on the nonverbal reasoning abilities of grade 5 students (Mémoire de maitrise). Nipissing University.

Plante, I., Théorêt, M. et Favreau, O. E. (2009). Student gender stereotypes: contrasting the perceived maleness and femaleness of mathematics and language. Educational psychology, 29(4), 385-405. http://dx.doi.org/10.1080/01443410902971500

Poirier, L. (2001). Enseigner les maths au primaire. Notes didactiques. Montréal, QC : ERPI. 
Pólya, G. (1945). How to solve it: a new aspect of mathematical method. Princeton, NJ : Princeton University Press.

Rajotte, T. (2009). L'effet d'un programme scolaire d'enseignement des échecs sur le développement des habiletés en résolution de problèmes mathématiques et sur le sentiment d'appartenance des élèves de cinquième année du primaire (Mémoire de maitrise, Université du Québec à Rimouski). Repéré à

http://semaphore.uqar.ca/207/1/Thomas Rajotte novembre2009.pdf

Reusser, K. (1990). From the text to situation to equation: Cognitive simulation of understanding and solving mathematical word problems. Dans H. Mandl, E. De Corte, S. N. Bennett et H. F. Friedrich (dir.), Learning and instruction: European Research in an International Context (vol. 2, p. 477-498). Oxford : Pergamon Press.

Royer, É. (2010). Leçons d'éléphants : pour la réussite des garçons à l'école. Québec, QC : École et comportement.

Scarano, C. F. (2014). Perflles motivacionales de niños que participan del Programa Ajedrez en la Escuela (Thèse de doctorat, Universidad de la República Uruguay). Repéré à

http://sifp1.psico.edu.uy/sites/default/files/Trabajos\%20finales/\%20Archivos/tfg camila freire.pdf

Thériault, J. (2006). Pour mieux accompagner les garçons à l'école : miser sur des différences liées au genre. Montréal, QC : Organisation mondiale pour l'éducation préscolaire.

Vallerand, R. J. et Hess, U. (2000). Méthodes de recherche en psychologie. Boucherville, QC : Gaëtan Morin.

Verschaffel, L. et De Corte, E. (2008). La modélisation et la résolution des problèmes d'application : de l'analyse à l'utilisation efficace. Dans M. Crahay, L. Verschaffel, E. De Corte et J. Grégoire (dir.), Enseignement et apprentissage des mathématiques. Que disent les recherches psychopédagogiques? (p. 153-176). Bruxelles : De Boeck. http://dx.doi.org/10.3917/dbu.craha.2008.01.0153

Verschaffel, L., Greer, B. et De Corte, E. (2000). Making sense of word problems. Lisse : Swets \& Zeitlinger.

Voyer, D. et Goulet, M.-P. (2013). La compréhension de problèmes écrits d'arithmétique au regard de l'habileté en lecture d'élèves de sixième année (11 ans). Revue des sciences de l'éducation, 39(3), 449-513. http://dx.doi.org/10.7202/1026310ar

Wai, J., Cacchio, M., Putallaz, M. et Makel, M. C. (2010). Sex differences in the right tail of cognitive abilities: A 30 year examination. Intelligence, 38(4), 412-423. http://dx.doi.org/10.1016/j.intell.2010.04.006

\section{Pour citer cet article}

Voyer, D., Rousseau, M., Rajotte, T., Freiman, V. et Cabot-Thibault, J. (2018). Le jeu d'échecs dans les classes du primaire : un moyen ciblé pour développer les habiletés des garçons en résolution de problèmes. Formation et profession,

26(3) 94-108. http://dx.doi.org/10.18162/fp.2018.440 